\title{
Effects of cryopreservation on the human sperm acrosome and its response to A23187
}

\author{
E. A. McLaughlin, W. C. L. Ford* and M. G. R. Hull \\ University of Bristol Department of Obstetrics and Gynaecology, St Michael's Hospital, \\ Bristol BS2 8EG, UK
}

\begin{abstract}
The proportion of human spermatozoa from 28 ejaculates to lose their acrosomes during cryopreservation was measured and correlated with the number that became immotile or lost the integrity of their plasma membrane. The ability of washed spermatozoa to acrosome react in response to A23187 before and after cryopreservation was compared. Motility was assessed by time-lapse photography; intact acrosomes were stained with fluorescein conjugated Pisum sativum agglutinin and dead spermatozoa were stained with bisbenzimide (H33258). Twenty-four per cent of spermatozoa lost their acrosomes during freezing and thawing, but the number that did so was not correlated with the number that became immotile or non-viable. Frozen spermatozoa exhibited fewer spontaneous acrosome reactions than did fresh spermatozoa ( 5 versus $13 \%$ after $4 \mathrm{~h}$ ), but they responded to A23187 in a similar way. Although frozen spermatozoa were significantly more likely to die during the incubation, the data do not suggest that degenerative acrosome loss had a major influence on the results. In the hamster egg test frozen-thawed spermatozoa achieved more penetrations

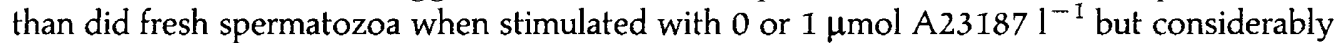
fewer when stimulated with $4 \mu \mathrm{mol}$ A23187 $\mathrm{l}^{-1}$. The following conclusions were made. First, cryopreservation damage to the acrosome, the plasma membrane and the flagellum can occur independently. Second, acrosome function is maintained after cryopreservation as long as the organelle remains mechanically intact. Third, some spermatozoa that lose their acrosomes during cryopreservation remain viable and can fuse with zona-free hamster eggs.
\end{abstract}

\section{Introduction}

More and more childless couples are requesting donor insemination, but to control the spread of human immunodeficiency virus (HIV) it is now mandatory to use frozen semen. More semen donors are needed to meet the demand, but many are rejected because their spermatozoa fail to survive cryopreservation (Schroeder-Jenkins and Rothman, 1989; Federation CECOS, 1989). Even when donors meet the usual criterion that $>60 \%$ of motile spermatozoa should survive freezing, the fecundity of the cryopreserved spermatozoa is lower than that achieved by fresh semen (Richter et al., 1984; Bordson el al., 1986; see Watson, 1990). Improved methods for cryopreservation of human spermatozoa might enhance the fertility of frozen semen and allow semen from a wider range of men to be used. However the development of these methods requires more detailed knowledge of the functional damage sustained by the spermatozoa during freezing and thawing.

Cryopreservation causes a decline in both the number of motile spermatozoa and their velocity (McLaughlin et al., 1992), but there is also extensive damage to the acrosome which

${ }^{*}$ Correspondence.

Received 21 August 1992. may contribute to the loss of fertility. After cryopreservation many spermatozoa have swollen or broken acrosomes and a decreased content of acrosin plus proacrosin (Pederson and Lebech, 1971; Mack and Zaneveld, 1987; Centola et al., 1990; Cross and Hanks, 1991). Much of the damage may occur during thawing (Woolley and Richardson, 1978) and physical damage to the acrosome may be secondary to cell death (Cross and Hanks, 1991). Cryopreserved spermatozoa achieve their maximum penetration of zona-free hamster eggs after a considerably shorter period of capacitation than do fresh spermatozoa (Critser et al., 1987). This finding suggests that frozen-thawed spermatozoa capacitate and acrosome react more quickly than do fresh spermatozoa. Spermatozoa that undergo the acrosome reaction prematurely might not be able to fertilize a zona-intact egg (Liu and Baker, 1990). However, this remains controversial because acrosome-reacted human spermatozoa can bind to the zona pellucida as well as acrosome intact spermatozoa (Morales et al., 1989).

The objectives of the present experiments were first, to measure damage to the acrosome during cryopreservation in ejaculates with a range of cryosurvival characteristics and second, to discover whether cryopreservation affects the ability of the surviving spermatozoa to acrosome react in response to graded doses of a calcium ionophore. 


\section{Materials and Methods}

\section{Semen collection and preparation}

Twenty-eight ejaculates (1-3 per donor) were collected from a group of 14 donors whose semen had a range of cryosurvival characteristics (McLaughlin et al., 1992). The semen was allowed to liquefy and a small sample was removed for standard seminal analysis. About a third of the ejaculate was washed three times by centrifugation in Biggers, Whitten and Whittingham buffer (BWW) (Aitken, 1983) $(300 \mathrm{~g}, 5 \mathrm{~min}$ ) and resuspended in the same medium $\left(20 \times 10^{6}\right.$ spermatozoa $\mathrm{ml}^{-1}$ ). The remainder of the ejaculate was mixed with an equal volume of glycerol-egg yolk-citrate cryopreservative buffer (GEYC), aspirated into $0.25 \mathrm{ml}$ plastic straws and frozen in a Nicool LM-10 semi-programmable freezer (McLaughlin et al., 1990). The straws were stored in liquid nitrogen until required. Semen was thawed by standing the straws on the bench top for $20 \mathrm{~min}$. A small sample was removed for semen analysis to assess survival and the remainder was diluted by the dropwise addition of $1.8 \mathrm{ml} \mathrm{BWW}$ per $0.25 \mathrm{ml}$ semen over a period of $5 \mathrm{~min}$. The spermatozoa were washed three times in BWW and were tested in the same way as fresh spermatozoa.

\section{Effect of $A 23187$ on the acrosome reaction}

A23187 (free acid) (Boehringer Mannheim GMBH, Lewes) was stored as a $50 \mathrm{mmol}^{-1}$ stock solution in anhydrous dimethylsulfoxide. $100 \mu \mathrm{mol}$ A23187 $1^{-1}$ was prepared by mixing $5 \mu \mathrm{l}$ of $50 \mathrm{mmol}$ A23187 $\mathrm{l}^{-1}$ with $2.495 \mathrm{ml}$ BWW with vigorous agitation and working solutions were prepared by diluting this to the desired concentration. All the experiments were done with ionophore from the same batch and the stock solution was not diluted until immediately before use.

Portions $(400 \mu \mathrm{l})$ of sperm suspension were diluted with an equal volume of $0,1,2,4$ or $8 \mu \mathrm{mol} \mathrm{A23187} \mathrm{(free} \mathrm{acid)} 1^{-1}$ in BWW and incubated at $37^{\circ} \mathrm{C}$ under $95 \%$ air : $5 \% \mathrm{CO}_{2}$ for $1 \mathrm{~h}$; the spermatozoa were then washed and resuspended in BWW at a concentration of $10 \times 10^{6} \mathrm{ml}^{-1}$ before they were incubated for a further $3 \mathrm{~h}$ at $37^{\circ} \mathrm{C}$ under $95 \%$ air: $5 \% \mathrm{CO}_{2}$. Samples for motility measurement by time-lapse photography (Rees $e t$ al., 1990) and the assessment of acrosomal status and vital staining (see below) were taken from the freshly resuspended spermatozoa $(0 \mathrm{~h})$, after incubation for $1 \mathrm{~h}$ with A23187 $(1 \mathrm{~h})$ and after the final incubation for $3 h(I+3 h)$.

\section{Vital staining and assessment of acrosome status}

Dead spermatozoa were stained with H33258 (bisbenzimide) (Boehringer Mannheim GMBH) and acrosomes were stained with fluorescein conjugated Pisum sativum agglutinin (FITCPSA) (Vector Laboratories Ltd, Peterborough) as described by Cross et al. (1986). The original authors observed the fluorescence of both H33258 and FITC-PSA simultaneously and could see whether individual acrosome reacted spermatozoa were viable or not. We could not achieve this with the equipment at our disposal and we were compelled to score the viability and the acrosome status of spermatozoa separately.
Consequently we could only infer whether acrosome reactions observed under the various conditions were viable from the frequency of dead spermatozoa in the population. Slides were examined with an Olympus BH2-LF microscope. An UGI excitation filter and a DM 400 dichroic mirror with a built-in $420 \mathrm{~nm}$ barrier filter were used to observe $\mathrm{H} 33258$ fluorescence. A BP490 excitation filter and a DM 500 dichroic mirror with a built-in $515 \mathrm{~nm}$ and supplementary $530 \mathrm{~nm}$ barrier filter were used to observe fluorescence. Duplicate slides were examined for each experimental condition. At least 200 spermatozoa were scored on each slide. Live/dead status was measured before acrosome status but the whole process was completed $<2 \mathrm{~h}$ after staining with FITC-PSA. A spermatozoon was taken to be acrosome intact if $\geq 50 \%$ of the anterior head was stained with FITC-PSA, otherwise it was regarded as acrosome reacted (Liu and Baker, 1988).

\section{Zona-free hamster egg test}

Six ejaculates from different donors were subjected to a hamster test before and after cryopreservation. The spermatozoa were prepared and incubated with ionophore for $1 \mathrm{~h}$ as described above and they were then added to zona-free hamster oocytes and incubated for $3 \mathrm{~h}$ (Ford et al., 1991). The oocytes were scored under phase contrast ( $\times 400$ magnification) and the number of decondensed sperm heads per egg was recorded. The data were converted to the values that would have been expected for a constant concentration of motile spermatozoa of $5 \times 10^{6} \mathrm{ml}^{-1}$ (Aitken and Elton, 1984).

\section{Statistical analysis}

The effects of cryopreservation on the acrosome status, viability and motility of the spermatozoa were tested with a one-way analysis of variance using the MINITAB statistics package (Minitab Inc., State College, Pennsylvania).

In the experiments to study the effect of A23187 on the acrosome reaction of fresh and frozen spermatozoa, analysis of the data was complicated because the frozen sperm populations contained fewer acrosome-intact sperm capable of responding to the ionophore. To overcome this problem, the data were expressed as a percentage of the spermatozoa with acrosome intact at zero time that underwent an acrosome reaction during the incubation, i.e. $A R=A R t-A R o /(100-A R o)$ where $A R o$ and $A R t$ represent the percentage of the sperm population which was acrosome reacted at zero time and after incubation, respectively. For similar reasons the results of the observations of the percentages of the spermatozoa that were viable or progressively motile were transformed according to the equation $\chi=\chi_{0}-X_{t} / \chi_{0}$, where $\chi_{0}$ and $X_{t}$ represent the percentages of the sperm population that were viable or progressively motile at zero time or after incubation, respectively. Data for sperm velocity and lateral head displacement were not transformed. The statistical significance of the effects of the treatment was tested with a three-way analysis of variance. Calculations were performed using the GLM procedure of the SAS statistics package (SAS Institute Inc., Cary). 
Table 1. Effect of cryopreservation on the motility, viability and acrosome integrity of human spermatozoa

\begin{tabular}{|c|c|c|c|c|c|}
\hline & \multirow[b]{2}{*}{$\begin{array}{l}\% \text { Acrosome } \\
\text { reacted }\end{array}$} & \multirow[b]{2}{*}{$\%$ Live } & \multicolumn{3}{|c|}{ Motility } \\
\hline & & & $\%$ Motile & $\begin{array}{l}\text { Velocity } \\
\left(\mu \mathrm{m} \mathrm{s}^{-1}\right)\end{array}$ & $\begin{array}{c}\text { Lateral head } \\
\text { displacement }(\mu \mathrm{m})\end{array}$ \\
\hline Fresh & $31 \pm 1.2$ & $86 \pm 1.3$ & $51 \pm 2.5$ & $69 \pm 2.0$ & $4.4 \pm 0.14$ \\
\hline Frozen & $55 \pm 1.6^{*}$ & $53 \pm 1.2^{*}$ & $28 \pm 2.3^{*}$ & $51 \pm 1.4^{*}$ & $3.6 \pm 0.14^{*}$ \\
\hline
\end{tabular}

Spermatozoa from fresh or frozen semen were washed three times and examined immediately after resuspension in Biggers, Whitten and Whittingham medium. Motility measurements were made by time-lapse photography at $37^{\circ} \mathrm{C}$; viability was assessed by the exclusion of bisbenzimide and acrosome status by staining with Fluorescein conjugated Pisum sativum agglutinin. Data are means $\pm \operatorname{SEM}, n=28$. ${ }^{*}$ Significant effect of freezing (analysis of variance) $P<0.0005$.

\section{Results}

Effect of cryopreservation on acrosome integrity, viability and motility

Freezing and thawing caused a considerable increase in the percentage of spermatozoa that lacked intact acrosomes. It also produced large decreases in the percentages of spermatozoa that were viable or progressively motile and reduced the velocity and lateral head displacement of the motile spermatozoa. All these changes were statistically significant $(P<0.0005)$ (Table 1). On average, similar proportions of spermatozoa lost their acrosomes, became permeable to bisbenzamide or became immotile. However, there was no significant correlation between any of these parameters or between the extents to which they changed.

\section{Effect of cryopreservation on the response of the spermatozoa to} A23187

A.23187 produced a dose-dependent increase in the number of spermatozoa that acrosome reacted during the $1 \mathrm{~h}$ stimulation period. The proportion of spermatozoa that underwent an acrosome reaction during this time was the same in fresh and in frozen spermatozoa (Table 2). Further spermatozoa exhibited acrosome reactions during the subsequent $3 \mathrm{~h}$ incubation but the numbers which did so were less clearly dependent on the concentration of A23187. Fewer frozen than fresh spermatozoa underwent the acrosome reaction during the $3 \mathrm{~h}$ incubation, except when they had been exposed to $4 \mu \mathrm{mol}$ A23187 $\mathrm{I}^{-1}$. This was responsible for a significant effect of freezing $(P<0.05)$ and a possible interaction between freezing and incubation time $(P=0.064)$ on the acrosome reaction (Table 4$)$. There was no significant interaction between the effects of freezing and of $A 23187$ on the acrosome reaction.

A23187 also caused significant and dose-related decreases in the proportions of motile and viable spermatozoa during the $1 \mathrm{~h}$ incubation. These deleterious effects continued in the $3 \mathrm{~h}$ incubation after the spermatozoa had been washed free of the ionophore (Tables 2,4$)$. There was a significant $(P<0.001)$ interaction of incubation time and A23187 for both parameters (Table 4), reflected in poor long-term survival of spermatozoa that had been exposed to the higher ionophore concentrations (Table 2). Compared to fresh spermatozoa, frozen spermatozoa exhibited a greater reduction in the number of progressively motile cells and larger decreases in the average velocity and lateral head displacement during the incubation (Table 3). However, the sensitivity of their motility parameters to the toxic effects of A23187 was similar (Tables 2-4). Significantly more frozen than fresh spermatozoa lost their membrane integrity during the experiment $(P<0.01$, Table 4$)$, but the adverse effects of A23187 and incubation time on viability were broadly similar in both fresh and frozen spermatozoa and no significant interactions between freezing and A23187 or incubation time were detected (Table 4).

\section{The zona-free hamster egg test}

Generally $1 \mu \mathrm{mol} \mathrm{A23187} 1^{-1}$ produced a small increase and

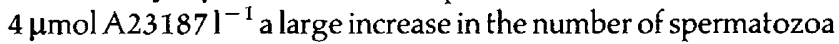
that penetrated the zona-free hamster eggs. In the presence of 0 or $1 \mu \mathrm{mol} A 23187 \mathrm{I}^{-1}$, the frozen spermatozoa achieved as many or more penetrations as fresh spermatozoa in 5 of 6 experiments but in the presence of $4 \mu \mathrm{mol} \mathrm{A} 231871^{-1}$ the situation was reversed and they achieved fewer penetrations in the same proportion of experiments (based on values corrected to $5 \times 10^{6}$ spermatozoa $\mathrm{ml}^{-1}$ ). The poor performance of frozen spermatozoa exposed to $4 \mu \mathrm{mol}$ A23187 $\mathrm{l}^{-1}$ was particularly marked in semen that survived freezing poorly and only one such sample achieved any penetrations at all under these conditions (Table 5). Otherwise the performance in the test of spermatozoa from samples with poor survival was within the same range as that of spermatozoa from semen with good survival, although at its lower end (Table 5). In the fresh spermatozoa, the number of acrosome reactions that occurred during the $1 \mathrm{~h}$ incubation with A23187 was correlated with the number of penetrations per hamster egg $(r=0.512, P<0.05$, $n=9$ ), but no such correlation could be demonstrated with frozen spermatozoa.

\section{Discussion}

One possible explanation of the absence of any significant correlation between the percentage of spermatozoa that lost 
Table 2. The effect of A23187 on the acrosome status, viability and motility of human spermatozoa prepared from fresh and cryopreserved semen

\begin{tabular}{|c|c|c|c|c|c|c|c|}
\hline \multirow[b]{3}{*}{ Parameter } & \multirow{3}{*}{$\begin{array}{l}\text { Incubation } \\
\text { (h) }\end{array}$} & \multirow[b]{3}{*}{ Spermatozoa } & \multicolumn{5}{|c|}{$\begin{array}{l}\text { Percentage of spermatozoa acrosome intact, } \\
\text { viable or progressively motile at zero time that } \\
\text { changed status during the incubation }\end{array}$} \\
\hline & & & \multicolumn{5}{|c|}{$\mathrm{A} 23187\left(\mu \mathrm{mol} !^{-1}\right)$} \\
\hline & & & 0 & 0.5 & 1.0 & 2.0 & 4.0 \\
\hline \multirow[t]{4}{*}{ Acrosome status } & 1 & Fresh & $6 \pm 2.0$ & $11 \pm 2.6$ & $17 \pm 2.6$ & $22 \pm 2.9$ & $31 \pm 3.2$ \\
\hline & & Frozen & $3 \pm 2.8$ & $9 \pm 2.8$ & $15 \pm 3.0$ & $25 \pm 3.5$ & $31 \pm 3.5$ \\
\hline & $1+3$ & Fresh & $13 \pm 2.2$ & $25 \pm 2.6$ & $32 \pm 3.0$ & $39 \pm 3.7$ & $44 \pm 3.2$ \\
\hline & & Frozen & $5 \pm 2.8$ & $16 \pm 3.3$ & $23 \pm 2.9$ & $33 \pm 3.0$ & $49 \pm 3.4$ \\
\hline \multirow[t]{4}{*}{ Viability } & $\mathbf{1}$ & Fresh & $4 \pm 3.5$ & $9 \pm 3.6$ & $6 \pm 3.6$ & $9 \pm 3.5$ & $16 \pm 3.1$ \\
\hline & & Frozen & $4 \pm 1.6$ & $9 \pm 2.1$ & $10 \pm 1.9$ & $15 \pm 1.8$ & $18 \pm 1.9$ \\
\hline & $1+3$ & Fresh & $7 \pm 3.0$ & $10 \pm 3.1$ & $12 \pm 3.1$ & $22 \pm 2.9$ & $34 \pm 3.2$ \\
\hline & & Frozen & $11 \pm 2.4$ & $14 \pm 1.9$ & $21 \pm 1.8$ & $28 \pm 2.1$ & $35 \pm 2.6$ \\
\hline \multirow[t]{4}{*}{ Motility } & 1 & Fresh & $2 \pm 7.2$ & $9 \pm 6.4$ & $5 \pm 6.7$ & $11 \pm 5.7$ & $22 \pm 5.2$ \\
\hline & & Frozen & $4 \pm 5.4$ & $5 \pm 6.6$ & $-1 \pm 7.5$ & $14 \pm 5.7$ & $24 \pm 6.9$ \\
\hline & $1+3$ & Fresh & $8 \pm 5.3$ & $12 \pm 5.9$ & $16 \pm 5.5$ & $35 \pm 4.1$ & $56 \pm 4.3$ \\
\hline & & Frozen & $7 \pm 6.5$ & $20 \pm 5.8$ & $27 \pm 6.6$ & $44 \pm 6.5$ & $68 \pm 4.9$ \\
\hline
\end{tabular}

The spermatozoa were washed three times and resuspended in Biggers, Whitten and Whittingham medium and incubated with A23187 for $1 \mathrm{~h}$ at $37^{\circ} \mathrm{C}$; they were then washed and incubated for a further $3 \mathrm{~h}$. The data are expressed as the percentage of the population that was acrosome intact, viable or motile, at zero time that changed status during the incubation. Zero time values are shown in Table 1 . All data are means \pm SEM, $n=28$. Details of the analysis of variance are shown in Table 4 .

Table 3. The effect of A23187 on the velocity and lateral head displacement of fresh and cryopreserved human spermatozoa

\begin{tabular}{|c|c|c|c|c|c|c|c|c|c|c|c|}
\hline \multirow[b]{2}{*}{ Parameter } & \multirow{2}{*}{$\begin{array}{c}\text { Incubation } \\
\text { (h) }\end{array}$} & \multirow[b]{2}{*}{ Spermatozoa } & \multicolumn{9}{|c|}{ A23187 $\left(\mu \mathrm{mol} \mathrm{l}^{-1}\right)$} \\
\hline & & & & 0 & & 0.5 & & 1.0 & & 2.0 & 4.0 \\
\hline \multirow{4}{*}{$\begin{array}{l}\text { Velocity } \\
\qquad\left(\mu \mathrm{m} \mathrm{s}^{-1}\right)\end{array}$} & 1 & Fresh & 69 & \pm 1.8 & 69 & \pm 1.7 & 67 & \pm 1.9 & 70 & \pm 1.8 & $66 \pm 1.9$ \\
\hline & & Frozen & 54 & \pm 1.6 & 53 & \pm 1.5 & 51 & \pm 1.6 & 51 & \pm 1.5 & $49 \underset{(26)}{ \pm} 1.7$ \\
\hline & $1+3$ & Fresh & 73 & \pm 2.1 & 72 & \pm 2.2 & 69 & \pm 2.1 & 62 & \pm 2.0 & $55 \pm 2.3$ \\
\hline & & Frozen & 52 & \pm 2.0 & 51 & $\underbrace{ \pm 2.0}_{(27)}$ & 46 & \pm 1.8 & 45 & $\underset{(23)}{ \pm} 1.9$ & $42 \underset{(17)}{ \pm 2.4}$ \\
\hline \multirow{4}{*}{$\begin{array}{l}\text { Lateral head } \\
\text { displacement } \\
(\mu \mathrm{m})\end{array}$} & 1 & Fresh & \multirow{2}{*}{\multicolumn{2}{|c|}{$\begin{array}{l}4.6 \pm 0.14 \\
3.6 \pm 0.11\end{array}$}} & \multirow{2}{*}{\multicolumn{2}{|c|}{$\begin{array}{l}4.7 \pm 0.17 \\
3.7 \pm 0.10\end{array}$}} & \multirow{2}{*}{\multicolumn{2}{|c|}{$\begin{array}{l}4.5 \pm 0.15 \\
3.6 \pm 0.08\end{array}$}} & \multirow{2}{*}{\multicolumn{2}{|c|}{$\begin{array}{l}4.6 \pm 0.17 \\
3.7 \pm 0.11\end{array}$}} & \multirow{2}{*}{$\begin{array}{c}4.5 \pm 0.17 \\
3.8 \pm 0.13 \\
(26)\end{array}$} \\
\hline & & Frozen & & & & & & & & & \\
\hline & $1+3$ & Fresh & \multirow{2}{*}{\multicolumn{2}{|c|}{$\begin{array}{l}4.8 \pm 0.15 \\
4.0 \pm 0.13\end{array}$}} & \multirow{2}{*}{\multicolumn{2}{|c|}{$\begin{array}{c}4.9 \pm 0.17 \\
3.8 \pm 0.13 \\
(27)\end{array}$}} & \multirow{2}{*}{\multicolumn{2}{|c|}{$\begin{array}{l}4.8 \pm 0.16 \\
4.1 \pm 0.14\end{array}$}} & \multirow{2}{*}{\multicolumn{2}{|c|}{$\begin{array}{c}4.9 \pm 0.20 \\
4.0 \pm 0.18 \\
(23)\end{array}$}} & \multirow{2}{*}{$\begin{array}{c}4.9 \pm 0.20 \\
4.3 \pm 0.22 \\
(17)\end{array}$} \\
\hline & & Frozen & & & & & & & & & \\
\hline
\end{tabular}

Experimental details are given in Table 2 and the analysis of variance is described in Table 4. Data are means \pm SEM, $n=28$ unless stated otherwise.

their acrosomal cap during cryopreservation and the percentage that died or became immotile is that damage to the acrosome, the flagellum and plasma membrane can occur independently. If this is so, frozen-thawed semen would contain a population of viable acrosome-reacted spermatozoa. Technical difficulties prevented us from confirming this by direct observation, but in cryopreserved semen the proportion of spermatozoa that was acrosome reacted was always greater than the proportion 
Table 4. Analysis of variance of the effects of A23187 and incubation time on different parameters of fresh and cryopreserved human spermatozoa

\begin{tabular}{|c|c|c|c|c|c|c|}
\hline \multirow[b]{3}{*}{ Source } & \multirow[b]{3}{*}{$d f$} & \multicolumn{5}{|c|}{ F Ratio } \\
\hline & & \multicolumn{5}{|c|}{ Parameter } \\
\hline & & $\begin{array}{c}\% \text { Acrosome } F \\
\text { reacted }\end{array}$ & $\%$ Viable $F$ & $\%$ Motile $F$ & Velocity & $\begin{array}{l}\text { Lateral head } \\
\text { displacement }\end{array}$ \\
\hline Freezing $(F)$ & 1 & $5.90^{*}$ & $9.34^{* * *}$ & 1.99 & $417.62^{* * *}$ & $16.95^{* * *}$ \\
\hline Time & 1 & $65.21^{* * *}$ & $63.56^{* * *}$ & $55.43^{* * *}$ & $10.91^{* *}$ & $19.35^{* * *}$ \\
\hline$F \times$ Time & 1 & 3.44 & 1.40 & 2.65 & 1.14 & $<0.16$ \\
\hline A23187 & 4 & $70.42^{* * *}$ & $29.64^{* * *}$ & $25.56^{* * *}$ & $13.55^{* * *}$ & 0.24 \\
\hline$F \times A 23187$ & 4 & 1.34 & 0.85 & 0.23 & 0.71 & 1.74 \\
\hline Time $\times A 23187$ & 4 & 1.69 & $4.89^{* * *}$ & $5.27^{* * *}$ & $5.66^{* * *}$ & 0.86 \\
\hline$F \times$ Time $\times A 23187$ & 4 & 0.85 & 0.33 & 0.43 & 1.75 & 0.13 \\
\hline
\end{tabular}

$F$ Analysis based on the percentage change during the incubation (Table 2).

${ }^{*} P<0.05,{ }^{* *} P<0.01,{ }^{* *} P<0.001$. Error mean square had $540 \mathrm{df}$.

Table 5. Results of the hamster egg test for human spermatozoa from three donors whose semen survived cryopreservation well and from three donors whose semen survived poorly

\begin{tabular}{|c|c|c|c|c|c|c|}
\hline \multirow{4}{*}{$\begin{array}{l}\text { Percentage of } \\
\text { motile spermatozoa } \\
\text { in fresh semen } \\
\text { to survive freezing }\end{array}$} & \multicolumn{6}{|c|}{ Decondensed sperm heads per egg (corrected to $5 \times 10^{6}$ spermatozoa ml ${ }^{-1}$ ) } \\
\hline & \multicolumn{6}{|c|}{ A23187 $\left(\mu \mathrm{m} \mathrm{l}^{-1}\right)$} \\
\hline & \multicolumn{2}{|c|}{0} & \multicolumn{2}{|c|}{1} & \multicolumn{2}{|c|}{4} \\
\hline & Fresh & Frozen & Fresh & Frozen & Fresh & Frozen \\
\hline \multirow[t]{3}{*}{$>60$} & $0 \quad(0)$ & $0.7(0.6)$ & $1.4(1.3)$ & $0.5 \quad(1.25)$ & $17.5(14.6)$ & $11.1(23.1)$ \\
\hline & $2.9(3.6)$ & $3.8(2.7)$ & $1.8(1.4)$ & $4.3(4.3)$ & $45.0 \quad(39.5)$ & $6.3(9.0)$ \\
\hline & $0.1(0.2)$ & $2.8(3.9)$ & $1.1(0.8)$ & $2.8 \quad(5.5)$ & 16.1 & $3.6 \quad(9.0)$ \\
\hline \multirow[t]{3}{*}{$<40$} & $0.4(0.3)$ & $1.4(1.7)$ & $0.4(0.4)$ & $8.6(14.3)$ & $11.3(14.1)$ & $(0)$ \\
\hline & $0.1(0.1)$ & $0.1(0.3)$ & $0.5(0.4)$ & $0.2(0.5)$ & $4.3 \quad(3.6)$ & $0 \quad(0)$ \\
\hline & $0 \quad(0)$ & $0 \quad(0)$ & $3.5(2.9)$ & $0 \quad(0)$ & $19.5(244)$ & 0.2 (1.1) \\
\hline
\end{tabular}

The data expressed as the average number of decondensed sperm heads observed per egg with the number expected had the concentration of progressively motile spermatozoa been $5 \times 10^{6} \mathrm{ml}^{-1}$ (Aitken and Elton, 1984) shown in parentheses.

that was dead. An increase in the proportion of viable acrosome-reacted spermatozoa after cryopreservation has been demonstrated by simultaneous single wavelength measurement of viability and acrosome status (Centola et al., 1990). The presence of such cells could account for the observation that frozen spermatozoa achieved more penetrations than did fresh spermatozoa in the hamster egg test when A23187 was not used to promote acrosome reactions. It would also explain why cryopreserved spermatozoa appeared to capacitate more rapidly than did fresh spermatozoa (Critser et al., 1987). However, Cross and Hanks (1991) considered that acrosome loss during cryopreservation was associated with cell death. Their conclusion was based on the observation that on average more spermatozoa became immotile than lost their acrosomes during cryopreservation and that motile spermatozoa recovered by a stringent swim-up technique were largely acrosome intact. This finding cannot exclude the presence of viable acrosome-reacted spermatozoa of moderate motility.

Both acrosome-reacted and acrosome-intact spermatozoa can bind to the zona pellucida in humans (Morales et al., 1989), but fewer bind after acrosome reactions are induced by A23187 (Liu and Baker, 1990). Bearing in mind the requirement for spermatozoa to traverse the female reproductive tract, it is probable that acrosome-reacted spermatozoa inseminated into the cervix are less likely to fertilize than are normal intact spermatozoa. Damage to the acrosome during cryopreservation may therefore contribute to the loss of fertility.

The second objective of these experiments was to discover whether freezing and thawing affected the ability of apparently intact spermatozoa to acrosome react in response to graded doses of A23187. In our view this is a physiologically relevant stimulus because, as was first demonstrated in the mouse 
(Wassarman, 1987), human spermatozoa can be stimulated to acrosome react by the zona pellucida (Cross et al., 1989). In bull spermatozoa, a solubilized preparation of the zona pellucida stimulated a rise in the intracellular $\mathrm{Ca}^{2+}$ concentration and in $\mathrm{pH}$ which preceded the acrosome reaction (Florman et al., 1989). A23187 produces similar changes in intracellular ion concentrations and provides a realistic model for the physiological control process downstream of the receptor binding event. Cryopreservation does not affect the ability of spermatozoa to bind to the zona pellucida (Gamzu et al., 1992). A23187 was very effective in inducing acrosome reactions, but there was no significant interaction between A23187 concentration and freezing in this study. Within the resolution of this experiment the sensitivity of fresh and of frozen spermatozoa to A23187 is therefore similar. Frozen spermatozoa exhibited significantly fewer acrosome reactions than did fresh spermatozoa. This difference was greatest after $\mathrm{I}$ plus $3 \mathrm{~h}$ of incubation and the interaction between freezing and incubation time nearly achieved statistical significance at the $5 \%$ level. One simple explanation for these observations is that the incidence of spontaneous acrosome reactions was less in the frozen spermatozoa. This may have occurred because the spermatozoa that were ready to react were precipitated to do so by the stress of freezing and thawing. There was extensive toxicity of the A23187 and we cannot rule out the possibility that some of the acrosome reactions were degenerative. However, there is strong circumstantial evidence indicating that degenerative acrosome loss was not a major factor: (1) the difference in the loss of viability between fresh and frozen spermatozoa, although significant, was relatively small; (2) there was no correlation between the percentages of spermatozoa to acrosome react and to die; and (3) the frozen sperm preparations contained a significantly greater proportion of dead spermatozoa at the start of the experiment $(47 \%$ versus $14 \%$ in fresh preparations). If significant numbers of dead spermatozoa were undergoing degenerative acrosome reactions, it would be expected that a higher spontaneous acrosome rate would be observed in the frozen than in the fresh spermatozoa, whereas the reverse occurred. We consider it likely that spermatozoa succumbed to the toxic effect of A23187 after acrosome reacting rather than dying and then undergoing an acrosome reaction. By contrast with fresh spermatozoa, frozen spermatozoa that had been stimulated to acrosome react with $4 \mu \mathrm{mol}$ A23 187 $1^{-1}$ achieved relatively few penetrations in the hamster egg test. The data have been adjusted to take account of the differences in motility, and differences in the loss of viability during the incubation are too small to account for the large change in the penetration rate. Further factors not measured here must therefore contribute to the loss of fertility during freezing.

\section{References}

Aitken RJ (1983) The zona free hamster egg penetration test. In Male Infertility pp 75-86 Ed. TB Hargreave. Springer Verlag, Berlin

Aitken RJ and Elton RA (1984) Significance of Poisson distribution theory in analysing the interaction between human spermatozoa and zona-free hamster oocytes Journal of Reproduction and Fertility 72 311-321
Bordson BL, Ricci E, Dickey RP, Dunaway H, Taylor SN and Curole DW (1986) Comparison of fecundability with fresh and frozen semen in therapeutic donor insemination Fertility and Sterility $46466-469$

Centola GM, Mattox JH, Burde S and Leary JF (1990) Assessment of the viability and acrosome status of fresh and frozen-thawed human spermatozoa using single wavelength fluorescence microscopy Molecular Reproduction and Development 27 130-135

Critser JK, Arneson BW, Aaker DV, Huse-Benda AR and Ball GD (1987) Cryopreservation of human spermatozoa II Post-thaw chronology of motility and of zona-free hamster ova penetration Fertility and Sterility $\mathbf{4 7}$ 980-984

Cross NL and Hanks SE (1991) Effects of cryopreservation on human sperm acrosomes Human Reproduction 6 1279-1283

Cross NL, Morales P, Overstreet JW and Hanson FW (1986) Two simple methods for detecting acrosome-reacted human sperm Gamete Research 15 213-226

Cross NL, Morales P, Overstreet JW and Hanson FW (1989) Induction of the acrosome reaction by the human zona pellucida Biology of Reproduction $\mathbf{3 8}$ 235-243

Federation CECOS, Le Lannon D and Lansac J (1989) Artificial procreation with frozen donor semen: experience of French Federation CECOS Human Reproduction 4 757-761

Florman HM, Tombes RM, First NL and Babcock DF (1989) An adhesion associated agonist from the zona pellucida activates $G$ protein mediated elevations of internal $\mathrm{Ca}^{++}$and $\mathrm{pH}$ that mediate mammalian sperm acrosomal exocytosis Developmental Biology 135 133-146

Ford WCL, Rees JM, McLaughlin EA, Goddard RJ and Hull MGR (1991) The effect of A23187 concentration and exposure time on the outcome of the hamster egg penetration test International Journal of Andrology 14 127-139

Gamzu R, Homonnai ZT, Yogev L, Hiss Y, Yavetz H and Paz G (1992) Fresh and frozen-thawed human sperm bind in a similar pattern to the zona pellucida in the hemizona assay Fertility and Sterility 58 1254-1256

Liu DY and Baker HWG (1988) The proportion of human sperm with poor morphology but normal intact acrosomes detected with Pisum sativum agglutinin correlates with fertilization in vitro Fertility and Sterility $\mathbf{5 0}$ 288-293

Liu DY and Baker HWG (1990) Inducing the human acrosome reaction with a calcium ionophore A23187 decreases sperm-zona pellucida binding with oocytes that failed to fertilize in vitro Joumal of Reproduction and Fertility $\mathbf{8 9}$ 127-134

Mack SR and Zaneveld LJD (1987) Acrosomal enzymes and ultrastructure of unfrozen and cryotreated human spermatozoa Gamete Research 18 375-383

McLaughlin EA, Ford WCL and Hull MGR (1990) A comparison of the freezing of human semen in the uncirculated vapour above liquid nitrogen and in a commercial semi-programmable freezer Human Reproduction 5 724-728

McLaughlin EA, Ford WCL and Hull MGR (1992) Motility characteristics and membrane integrity of cryopreserved human spermatozoa Joumal of Reproduction and Fertility 95 527-534

Morales P, Cross NL, Overstreet JW and Hanson FW (1989) Acrosome intact and acrosome reacted human sperm can initiate binding to the zona pellucida Developmental Biology 133 385-392

Pederson H and Lebech PE (1971) Ultrastructural changes in the human spermatozoa after freezing for artificial insemination Fertility and Sterility $\mathbf{2 2}$ 125-133

Rees JM, Ford WCL and Hull MGR (1990) The effect of caffeine and pentoxifylline on the motility and metabolism of human spermatozoa Journal of Reproduction and Fertility 90 147-156

Richter MA, Haning RV and Shapiro S (1984) Artificial donor insemination: fresh versus frozen semen; the patient as her own control Fertility and Sterility $\mathbf{4 1}$ $277-280$

Schroeder-Jenkins MT and Rothman SA (1989) Causes of donor rejection in a sperm banking program Fertility and Sterility 51 903-906

Wassarman PM (1987) The biology and chemistry of fertilization Science 235 $553-560$

Watson PF (1990) Artificial insemination and the preservation of semen. In Marshall's Physiology of Reproduction Vol 2 (4th Edn) PP 747-869 Ed. GE Lamming. Churchill Livingstone, Edinburgh

Woolley DM and Richardson DW (1978) Ultrastructural injury to human spermatozoa after freezing and thawing Journal of Reproduction and Fertility 53 389-394 